\title{
MT1JP-mediated miR-24-3p/BCL2L2 axis promotes Lenvatinib resistance in hepatocellular carcinoma cells by inhibiting apoptosis
}

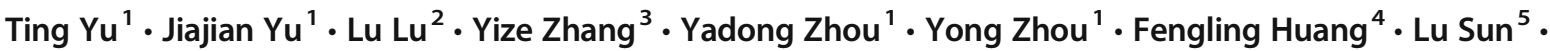 \\ Zhixian Guo ${ }^{5}$ - Guojun Hou ${ }^{6} \cdot$ Zihui Dong $^{3} \cdot$ Bibo Wang ${ }^{2}$
}

Accepted: 22 March 2021 / Published online: 11 May 2021

(C) The Author(s) 2021

\begin{abstract}
Purpose Lenvatinib is a long-awaited alternative to Sorafenib for first-line targeted therapy of patients with advanced hepatocellular carcinoma (HCC). However, resistance to Lenvatinib results in tumor progression and has become a major obstacle to improving the prognosis of HCC patients. Exploring the mechanisms underlying Lenvatinib resistance is considered essential for the treatment of advanced HCC.

Methods Lenvatinib resistant HCC (LR-HCC) cells were generated and potential long non-coding RNAs (Lnc-RNAs) upregulated in LR-HCC cells were identified by RNA sequencing. The effects of upregulated Lnc-RNAs were evaluated in vitro in cell models and in vivo in experimental animals using quantitative cell viability and apoptosis assays.

Results We found that Lnc-RNA MT1JP (MT1JP) was upregulated in LR-HCC cells and inhibited the apoptosis signaling pathway. In addition, we found that sponging of microRNA-24-3p by MT1JP released Bcl-2 like 2 (BCL2L2), an anti-apoptotic protein, thereby forming a positive-feedback loop. The role of this feedback loop was validated using rescue assays. Additionally, we found that upregulation of MT1JP and BCL2L2 impaired the sensitivity of HCC cells to Lenvatinib both vitro and vivo. Conclusions Our results suggest a novel molecular feedback loop between MT1JP and apoptosis signaling in Lenvatinib sensitive HCC cells.
\end{abstract}

Keywords Hepatocellular carcinoma $\cdot$ Lenvatinib $\cdot$ Sorafenib $\cdot$ Resistance $\cdot$ Apoptosis

\section{Introduction}

Hepatocellular carcinoma (HCC) is one of the most frequent and aggressive cancers and the third leading cause of cancerrelated death [1]. Approximately 700,000 new patients are diagnosed with HCC, annually. The development of HCC is tightly linked to sustained inflammation activated by either Hepatitis B virus (HBV) or Hepatitis C virus (HCV), alcohol abuse and/or metabolic disorders [2]. Despite clinical improvements that have been made, HCC patients are usually

Ting Yu, Jiajian $\mathrm{Yu}, \mathrm{Lu} \mathrm{Lu}$ and Yize Zhang contributed equally to this work.

Guojun Hou

Zihui Dong

Bibo Wang

1 Department of Hepatobiliary, Fuling Central Hospital of Chongqing City, Chongqing, China

2 Department of Medical Oncology, Jinling Hospital, School of Medicine, Nanjing University, Nanjing, China
3 Precision Medicine Center, Gene Hospital of Henan Province, The First Affiliated Hospital of Zhengzhou University, Zhengzhou, China

4 Department of Radiology, Fuling Central Hospital of Chongqing City, Chongqing, China

5 Department of Infectious Diseases, The First Affiliated Hospital of Zhengzhou University, Zhengzhou, China

6 The Third Department of Hepatic Surgery, Eastern Hepatobiliary Surgery Hospital, Second Military Medical University, Shanghai, China 
diagnosed with advanced stage disease. In addition, they frequently exhibit recurrences, resulting in five-year survival rates of $<20 \%$ [3]. Previous work has shown that hepatocellular carcinogenesis is a complex multi-step process, including genetic, epigenetic and cellular signaling alterations. As a result, HCCs extensively display heterogeneous molecular signatures [4]. As yet, however, our understanding of the molecular mechanisms underlying hepatocarcinogenesis is far from complete.

Since 2008, only Sorafenib, a tyrosine kinase inhibitor (TKI), has been approved by the food and drug administration (FDA) as first-line drug for unresectable HCC [5]. Although Regorafenib and Nivolumab were approved by the FDA as second-line treatments for Sorafenib-resistant HCC [6, 7], no other novel first-line drug was available for HCC until Lenvatinib (e7080), another TKI, was presented. In a randomized phase III clinical trial, it was found that Lenvatinib exhibited a non-inferior survival benefit compared to Sorafenib for untreated advanced HCC [8]. Lenvatinib has also been approved by the FDA for first-line treatment of HCC in August 2018.

Lenvatinib is an oral multi-kinase inhibitor that targets VEGF receptors, FGF receptors, PDGF receptor $\alpha$, RET and KIT to suppress their downstream signaling pathways, including targets shared by Sorafenib $[9,10]$. The clinical efficacy of Lenvatinib is well established now and has been found to extend HCC patient survival by 13.6 months, while for Sorafenib this was 12.3 months $[8,11]$. This modest survival benefit may, however, be lost due to drug resistance, as has been observed for Sorafenib [12]. Lenvatinib primarily affects angiogenesis, and additional studies implied that orally administered Lenvatinib exhibited anti-angiogenic activity in thyroid cancer, lung cancer and HCC [8, 10, 13]. In addition, anti-proliferative effects of Lenvatinib have been observed in different cancer cell types, both in vitro and in vivo $[14,15]$. Also, apoptosis-related caspase- 3 and caspase- 9 expression levels were found to be upregulated by Lenvatinib [16] and apoptotic rates to be increased in a Lenvatinib dose-dependent manner [15]. However, the mechanisms underlying Lenvatinib resistance (LR) are complicated and, as yet, largely unknown. Further investigations on the molecular basis of Lenvatinib resistance may shed light on the identification of new molecular targets to overcome this resistance.

Here, we generated two LR-HCC cell lines and performed RNA sequencing in these cell lines to systematically evaluate factors driving Lenvatinib resistance. We identified LncMT1JP (MT1JP) as a critical driver of Lenvatinib resistance in HCC. In addition, we found that MT1JP functions as a molecular sponge of miR-24-3p to inhibit apoptosis through the anti-apoptotic protein BCL2 Like 2 (BCL2L2). Our findings indicate that the MT1JP/miR-24-3p/BCL2L2 axis contributes to resistance of $\mathrm{HCC}$ cells to Lenvatinib and that targeting MT1JP-induced apoptosis may be a promising strategy to overcome TKI resistance in human HCC.

\section{Materials and methods}

\subsection{Cell culture and transfection}

HCC cell lines SMMC-7721 and Huh7 were purchased from the Shanghai Institute of Cell Biology, Chinese Academy of Sciences. Both cell lines were maintained in DMEM supplemented with $10 \%$ fetal bovine serum and incubated at $37^{\circ} \mathrm{C}$ in $5 \% \mathrm{CO}_{2}$. MT1JP overexpression plasmid (pcDNA-MT1JP), MT1JP siRNAs, miR-24-3p mimics and miR-24-3p inhibitor, BCL2L2 overexpression plasmid, BCL2L2 siRNAs as well as negative control oligos were synthesized by GenePharm (Shanghai, China). For transfection, SMMC-7721 and Huh7 cells at 60\%-80\% confluence were transfected with these plasmids using Lipofectamine 2000 (Invitrogen) according to the manufacturer's protocols.

\subsection{Establishment of Lenvatinib-resistant cell lines}

To determine the $\mathrm{IC}_{50}$ of SMMC-7721 and Huh7 cells to Lenvatinib treatment, the cells were incubated with different concentrations of Lenvatinib in 96-well plates, followed by cell viability measurements 3 days later. In order to establish Lenvatinib-resistant cell lines, SMMC-7721 and Huh7 cells were incubated with Lenvatinib (Selleck, S1164) at a concentration just below their $\mathrm{IC}_{50}$, and increased by $0.2 \mu \mathrm{M} / \mathrm{L}$ per week for 6 to 7 months. Two Lenvatinib-resistant cell lines were obtained, termed 7721-LR and Huh7-LR. These two Lenvatinib-resistant cell lines were maintained by continued culture in the presence of Lenvatinib.

\section{3 qRT-PCR assay}

Total RNA was isolated using Trizol reagent (Invitrogen) and reverse transcribed to cDNA using a TaqMan reverse transcription Kit (Applied Biosystems Life Technologies). qRTPCR was performed using a SYBR Green PCR Kit (Roche) on an ABI Prism 7300 Sequence Detection System (Applied Biosystems). The primers used are listed in Supplementary Table 1.

\subsection{Western blotting}

Total proteins were extracted using RIPA lysis buffer, and protein concentrations were determined using a BCA protein assay kit (Beyotime Biotechnology). The proteins $(40 \mu \mathrm{g})$ were separated on $12 \%$ SDS-polyacrylamide gels and transferred to PVDF membranes (Millipore). The membranes were incubated with the following primary antibodies: antiCaspase-3 (CST \#9662), anti-Cleaved Caspase-3 (CST \#9661), anti-PARP (CST \#9532), anti-Cleaved PARP (CST \#9548), anti-BCL2 (CST \#15071), purchased from Cell Signaling Technology, and anti-BCL2L2 (ab190952) and 
anti-GAPDH (ab181602) purchased from Abcam. GAPDH was used as loading control to normalize protein concentrations. Protein bands were visualized using an Odyssey Infrared Imaging system (LI-COR Biosciences USA).

\subsection{Cell viability and apoptosis assays}

Cell viability assays were performed in $96-$ well culture plates by seeding the cells at a density of $2 \times 10^{3}$ cells/well and incubation at $37^{\circ} \mathrm{C}$ in $5 \% \mathrm{CO}_{2}$ overnight. At the indicated time points, $10 \mu \mathrm{lCCK} 8$ solution (Beyotime Biotechnology) was added to each well and incubated for one hour, after which absorbance was measured at $450 \mathrm{~nm}$ using a plate reader. For Annexin V-FITC/PI double staining apoptosis detection, cells were collected and harvested, washed with cold PBS, adjusted to $1 \times 10^{6}$ cells $/ \mathrm{ml}$ in $1 \mathrm{x}$ binding buffer and stained with Annexin V-FITC and PI solution (BD Pharmingen, San Diego, CA, USA) for $15 \mathrm{~min}$ at room temperature in the dark. Finally, the stained cells were analyzed using flow cytometry (BD FACSCalibur, San Jose, CA, USA).

\subsection{TUNEL assay}

Apoptotic cell death was detected using a terminal deoxyribonucleotide transferase (TdT)-mediated dUTP nick-end labeling (TUNEL) detecting system (R\&D, AF835) as described previously [17].

\subsection{Transwell invasion assay}

A modified double chamber (Costar, Cambridge, NY, USA) was used to test cell invasion. $1 \times 10^{5}$ cells were seeded in the upper chamber in $100 \mu \mathrm{l}$ serum-free DMEM, and the lower chamber was filled with $600 \mu \mathrm{l}$ DMEM/10\% FBS. After incubation for $24 \mathrm{~h}$, the invaded cells were stained with crystal violet. Images were captured using a bright field microscope.

\subsection{In situ hybridization}

Double digoxigenin (DIG)-labeled locked nucleic acid probes for MT1JP (TTCCTGCTGAACTCACC, RNA-Tm $84^{\circ} \mathrm{C}$ ), and a scrambled sequence (GTGTAACACGTCTA TACGCCCA, RNA-Tm $87{ }^{\circ} \mathrm{C}$ ) as a negative control (Exiqon, Vedbaek, Denmark) were used according to the manufacturer's manual. Briefly, cells were fixed with $4 \%$ paraformaldehyde and incubated with Proteinase-K $(15 \mu \mathrm{g} / \mathrm{ml})$ for $10 \mathrm{~min}$ at $37^{\circ} \mathrm{C}$. After washing twice in phosphate buffered saline (PBS), the cells were dehydrated in ethanol, blocked in prehybridization buffer (3\% bovine serum albumin [BSA]) for $30 \mathrm{~min}$ at $55^{\circ} \mathrm{C}$ and incubated in hybridization buffer with probes (diluted at $1: 2000$ ) for $1 \mathrm{~h}$ at $55^{\circ} \mathrm{C}$. Next, the cells were washed with standard saline citrate buffer and blocked with $4 \%$ BSA for $1 \mathrm{~h}$ at room temperature. Positive signals were detected after overnight incubation with an anti-DIG primary mouse monoclonal antibody at $4{ }^{\circ} \mathrm{C}$, followed by incubation with fluorescein isothiocyanate (FITC)-conjugated antibodies. DAPI (4',6-diamidino-2phenylindole) was used to stain the cell nuclei. Finally, stained cells were evaluated using laser scanning confocal microscopy.

\subsection{Luciferase reporter assay}

A MT1JP fragment containing putative miR-24-3p target sites was amplified by PCR using 5'-CTCCTGCAAGAAGA GCTGC-3' and 5'-TGCAGCAAATGGCTCAGTA-3' primers, and cloned into the NaeI and HindIII sites of a pMIR-7 REPORT vector (Ambion). This reporter construct was named wild-type-MT1JP (WT-MT1JP). To generate a mutant reporter plasmid, we used a Generate site-directed mutagenesis system (Invitrogen) to introduce mutations into the putative miR-24-3p target sites of the wild-type-MT1JP vector. A mutant reporter (MUT-MT1JP) was constructed in which "GGCUCAGU" [71825078-718,250,786 nt] was converted into "CCGAGTCG". The reporter plasmid was transfected into cells using Lipofectamine 2000. To correct for transfection efficiency, an empty luciferase reporter vector without the miR-24-3p target was transfected in parallel. Luciferase activities in cells were expressed as ratios of the luciferase activity of the reporter vector with the miR-24-3p targeting sequence over the one without the targeting sequence. After $48 \mathrm{~h}$ of transfection with or without miR-24$3 p$ inhibitor, cells were harvested and luciferase activity was measured using a Dual-Luciferase Assay Kit (Promega, Madison, WI, USA).

\subsection{Xenograft tumor models}

NOD Scid Gamma (NSG) or BALB/c nude mice (4-6 weeks old) were purchased from the Animal Core Facility of Nanjing Medical University, housed under a standard 12-h light/dark cycle and fed a standard rodent chow diet in laminar flow cabinets under specific pathogen-free conditions. All procedures regarding animal handling were in accordance with the Guide for the Care and Use of Laboratory Animals published by the National Institutes of Health (NIH). Human studies were approved by the Ethics Boards of Fuling Central Hospital of Chongqing City and written informed consent was obtained from each patient. For xenografts, approximately $5 \times 10^{6}$ SMMC-7721 cells or transfected SMMC-7721 cells were subcutaneously implanted into the right flanks of nude mice (12 mice per group). For the establishment of patientderived xenograft (PDX) models, fresh surgical tumor tissues $\left(\mathrm{P}_{0}\right)$ were sectioned into $1-3 \mathrm{~mm}^{3}$ pieces and implanted subcutaneously into the flanks of female NSG mice. After 1 to 
3 months, PDX tumors $\left(\mathrm{P}_{1}\right)$ were transplanted $\left(\mathrm{P}_{2}-\mathrm{P}_{\mathrm{n}}\right.$ generations) under the skin of nude or NSG mice. When the tumors reached $\sim 100 \mathrm{~mm}^{3}$ they were assigned randomly to different groups ( $n=5$ /group). Xenograft tumor sizes were measured every 5 days and calculated by using the equation $\mathrm{V}\left(\mathrm{mm}^{3}\right)=$ (length $\mathrm{x}$ width $\left.{ }^{2}\right) / 2$. Mice that orally received Lenvatinib at concentrations of 0 to $100 \mathrm{mg} / \mathrm{kg}$ per day were maintained until the tumors grew to $50 \mathrm{~mm}^{3}$. All mice were sacrificed 30 days after the start of the Lenvatinib treatment, after which the tumor tissues were weighed and used for subsequent experiments.

\subsection{Immunohistochemistry (IHC)}

For immunohistochemistry (IHC) of paraffin sections, endogenous peroxide activity was quenched through $10 \mathrm{~min}$ incubation in $3 \% \mathrm{H}_{2} \mathrm{O}_{2}$, after which non-specific binding was blocked with serum. The resulting sections were incubated with an antiKi67 (Abcam ab245113) antibody at a 1:100 dilution overnight. Subsequently, diluted biotinylated anti-rabbit $\operatorname{IgG}$ (Vectastain kit) was added to the sections and incubated for 30 min. Next, Vectastain ABC reagent (Vector) and 3, 30diaminobenzamidine (DAB) were used for color development. Counterstaining was performed using hematoxylin solution.

\subsection{Statistical analysis}

The data are expressed as mean \pm SEM. Statistical significance was assessed using Student's $t$ test. Overall survival distributions were estimated using Kaplan-Meier analysis. $P<0.05$ was considered to be statistically significant. All experimental data were analyzed using SPSS16.0 (IBM, New York, USA) and all results were obtained from at least three separate experiments.

\section{Results}

\subsection{Lenvatinib resistant HCC cells are refractory to Lenvatinib-induced growth inhibition and apoptosis}

We found that Lenvatinib exposure inhibited the viability (Fig. 1a) and promoted the apoptosis (Fig. 1b-c) of both SMMC-7721 and Huh7 cells, and resulted in a slight variation in migration (Fig. S1A-B). The apoptotic rates of SMMC7721 and Huh7 cells were 4.2- and 5.1-fold increased (14.24\% vs $3.35 \%$ and $11.98 \%$ vs $2.35 \%$ ) after exposure to $0.5 \mu \mathrm{M} / \mathrm{L}$ Lenvatinib, respectively (Fig. 1b). These results were supported by the expression of two key apoptotic proteins, caspase- 3 and PARP and an anti-apoptotic protein, Bcl2 , i.e., Lenvatinib downregulated $\mathrm{Bcl}-2$ and promoted the cleavage of caspase-3 and PARP (Fig. 1c).
Two Lenvatinib-resistant HCC cells lines, 7721-LR and Huh7-LR, were established by first determining the Lenvatinib $\mathrm{IC}_{50}$ values of SMMC-7721 and Huh7 cells (which were 0.42 and $0.60 \mu \mathrm{M}$, respectively; Fig. 1a), and next incubation of the cells with gradually increasing concentrations of Lenvatinib for 6 to 7 months. The Lenvatinib-resistant properties of the LR-HCC cells were confirmed by comparing their viabilities with the parental HCC cells following incubation with various concentrations of Lenvatinib (Fig. 1d). After acquiring resistance, 7721-LR and Huh7-LR cells exhibited increased anti-apoptosis and higher migration capacities (Fig. 1e-g and S1C-D).

Even without Lenvatinib stimulation, the apoptotic rates of SMMC-7721 and Huh7 cells were 2.1- and 2.4-fold higher ( $5.25 \%$ vs $2.53 \%$ and $4.01 \%$ vs $1.70 \%$ ) than those of $7721-$ LR and Huh7-LR cells (Fig. S1E). After exposure to $0.5 \mu \mathrm{M}$ Lenvatinib, the apoptotic rates of 7721-LR and Huh7-LR cells decreased by 1.9 - and 2.0 -fold ( $7.66 \%$ vs $14.31 \%$ and $6.06 \%$ vs $12.29 \%$ ) compared to those of SMMC-7721 and Huh7 cells (Fig. S1E). These results were confirmed using a TUNEL assay (Fig. 1e-f). In addition, we found that acquiring resistance to Lenvatinib led to inhibition of the apoptosis pathway and its downstream factors including caspase-3 and PARP (Fig. 1g).

\subsection{Lenvatinib-resistant HCC cells overexpress Lnc MT1JP}

RNA sequencing (RNA-seq) was used to asses transcriptional changes induced by Lenvatinib resistance in the respective HCC cell lines (Fig. S2A). Subsequent GO analysis revealed that $22(6.7 \%)$ up-regulated long non-coding RNAs (LncRNAs) differentially expressed in 7721-LR cells relative to parental SMMC-7721 cells were also differentially expressed in Huh7-LR cells relative to parental Huh7 cells (Fig. 2a-b). Expression alterations of the top 10 of these 22 genes in the two Lenvatinib-resistant HCC cell lines were confirmed by qRT-PCR (Fig. S2B-C). To reveal the functions of these Lnc-RNAs, the top Lnc-RNAs were exogenously overexpressed in both SMMC-7721 and Huh7 cells after which their viability after exposure to Lenvatinib was assessed. The results obtained significantly pointed to a critical role of Lnc-MT1JP (MT1JP) on HCC cell viability (Fig. 2c). In addition we found that, after incubation for $48 \mathrm{~h}$, the expression of MT1JP increased with increasing concentrations of Lenvatinib both in SMMC-7721 and Huh7 cells (Fig. 2d). We next examined the intracellular location of MT1JP by assessing its expression in nuclear and cytoplasmic fractions of SMMC-7721 and Huh7 cells using in situ hybridization with MT1JP-specific probes. The results indicated that $75 \%-80 \%$ of MT1JP was located in the cytoplasmic fractions of the HCC cells (Fig. 2e and S2D). 
a

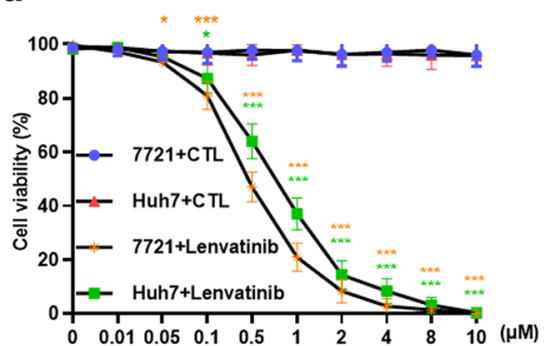

b

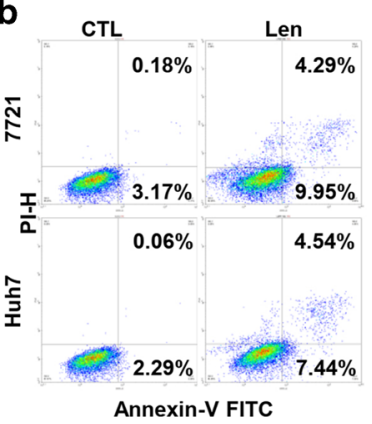

C

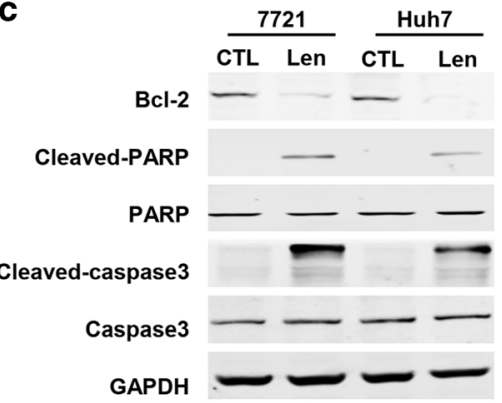

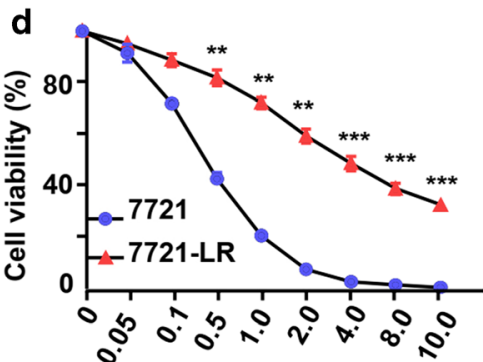

Lenvatinib $(\mu \mathrm{M})$

e
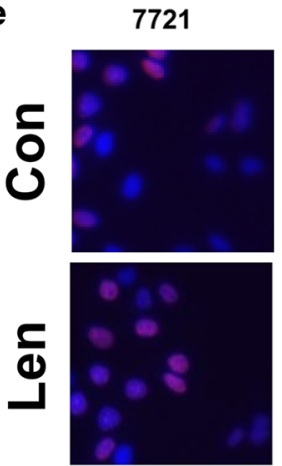

7721-LR
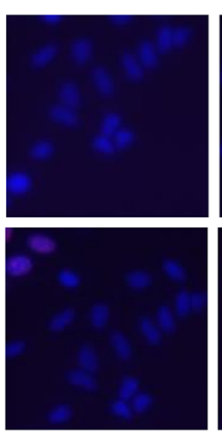

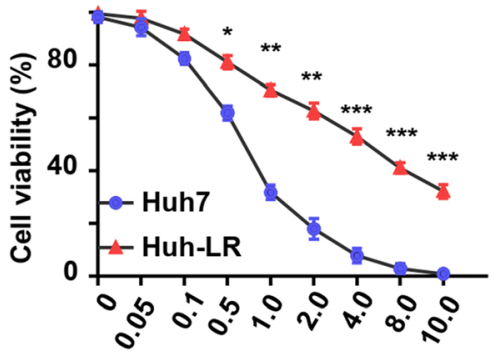

Lenvatinib $(\mu \mathrm{M})$
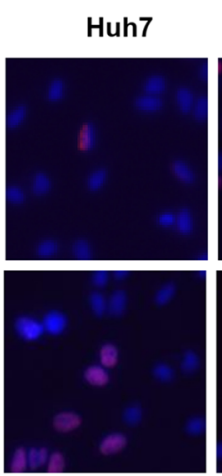

TUNE
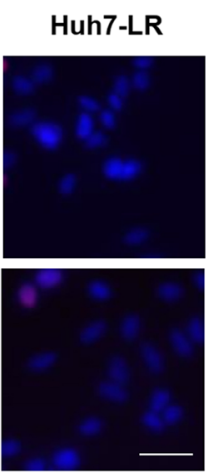

DAPI

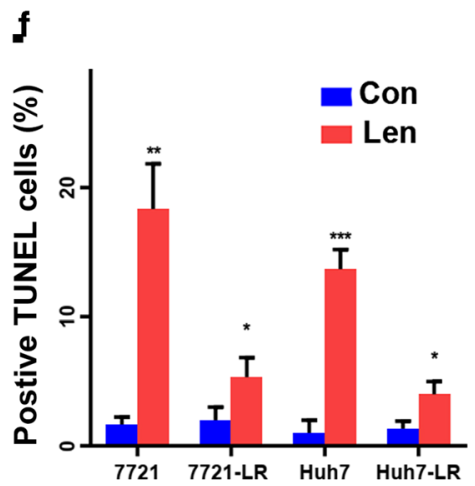

g

$$
\frac{7721}{\text { Con Len Con Len }} \frac{\text { 7721-LR }}{\text { Con Len Con Len }}
$$

$\mathrm{Bcl}-2$

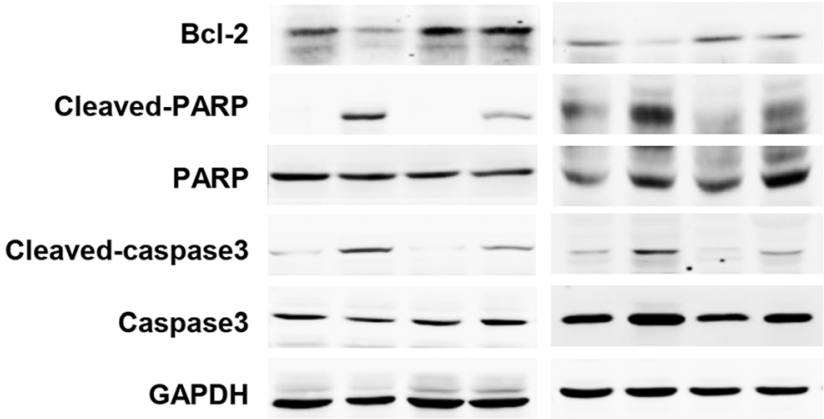

Fig. 1 Lenvatinib-resistant $\mathrm{HCC}$ cells are refractory to Lenvatinibinduced growth inhibition and apoptosis. a. HCC cell lines SMMC7721 and Huh7 were incubated with increasing concentrations of Lenvatinib for $48 \mathrm{~h}$. Cell viability (\%) was compared with corresponding untreated cells $(n=4)$. b-c. HCC cell lines were incubated with or without $0.5 \mu \mathrm{M} / \mathrm{L}$ Lenvatinib for $48 \mathrm{~h}$. The cells were analyzed cytometrically to detect apoptosis (b), the rates of apoptosis were labeled, and cell lysates were immunoblotted (c). For Western blotting, band densities were normalized to GADPH. d. Lenvatinib-resistant HCC cells (7721-LR, Huh7-LR) were incubated with Lenvatinib for $48 \mathrm{~h}$ after which cell viabilities detected by CCK 8 assay were calculated $(n=4)$. e-g. Apoptosis was detected in 7721-LR, Huh7-LR and parental 7721 and Huh7 cells, which were incubated with or without Lenvatinib (Len and Con). Results of TUNEL (e), its quantity (f) and Western blots $(\mathbf{g})$ are presented. Scale bars: $10 \mu \mathrm{m}$. Compared using Student's t test, $* p<0.05, * * p<0.01$, $* * * p<0.001$ 
Fig. 2 LINC-MT1JP is upregulated in Lenvatinibresistant $\mathbf{H C C}$ cells. a-b. Venn diagram showing overlap of differentially upregulated expression of genes in LR-7721 cells relative to control versus LRHuh7 cells relative to control. Details of the identified candidate genes are listed in (b). c. SMMC7721 or Huh7 cells were subjected to overexpression of these 10 candidate genes after which cell viabilities (\%) were determined and compared with LR$\mathrm{HCC}$ cells treated with Lenvatinib $(n=3)$. d. qRT-PCR confirmation of Lnc-MT1JP (MT1JP) expression with increasing concentrations of Lenvatinib for $48 \mathrm{~h}(n=$ 4). e. Total RNA was extracted from nuclear and cytoplasmic fractions after which the expression of MT1JP was determined by qRT-PCR and normalized. U1 was used as internal nuclear control. GAPDH was used as a cytoplasmic control. Compared using Student's t test, $* p<0.05$, $* * p<0.01, * * * p<0.001$ a

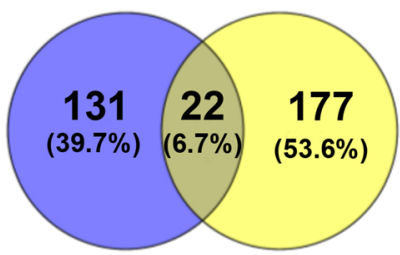

Upregulated LncRNA

in 7721-LR than 7721

Upregulated LncRNA

in Huh7-LR than Huh7 b

\begin{tabular}{|c|c|c|c|c|c|}
\hline & \multicolumn{2}{|c|}{ Fold } & \multicolumn{2}{|c|}{ Gene } & \multicolumn{2}{|c|}{ Fold } \\
\hline MT1JP & 7721 & Huh7 & & 7721 & Huh7 \\
\hline Lnc003391 & 4.16 & 3.09 & Lnc-TUC338 & 2.06 & 2.41 \\
\hline MALAT1 & 2.28 & 1.46 & Lnc-ROR & 3.17 & 1.44 \\
\hline Lnc-NEAT1 & 2.98 & 1.52 & Lnc01419 & 1.92 & 3.58 \\
\hline Lnc-DANCR & 3.70 & 1.35 & NPSR1-AS1 & 1.37 & 1.37 \\
\hline FOXD2-AS1 & 2.04 & 1.48 & Lnc-CASC15 & 1.62 & 5.16 \\
\hline Lnc-SNHG1 & 4.48 & 3.14 & Lnc-CRNDE & 1.59 & 3.09 \\
\hline Lnc-ARSR & 2.24 & 1.31 & Lnc-HCP5 & 3.29 & 2.16 \\
\hline Lnc-THOR & 1.51 & 1.97 & Lnc-FENDRR & 1.46 & 5.44 \\
\hline Lnc-GAS5 & 2.90 & 6.18 & Lnc00668 & 1.37 & 1.52 \\
\hline Lnc-HOXA13 & 1.41 & 3.52 & Lnc-LALR1 & 1.74 & 2.84 \\
\hline & & & & & 1.42 \\
\hline
\end{tabular}

C
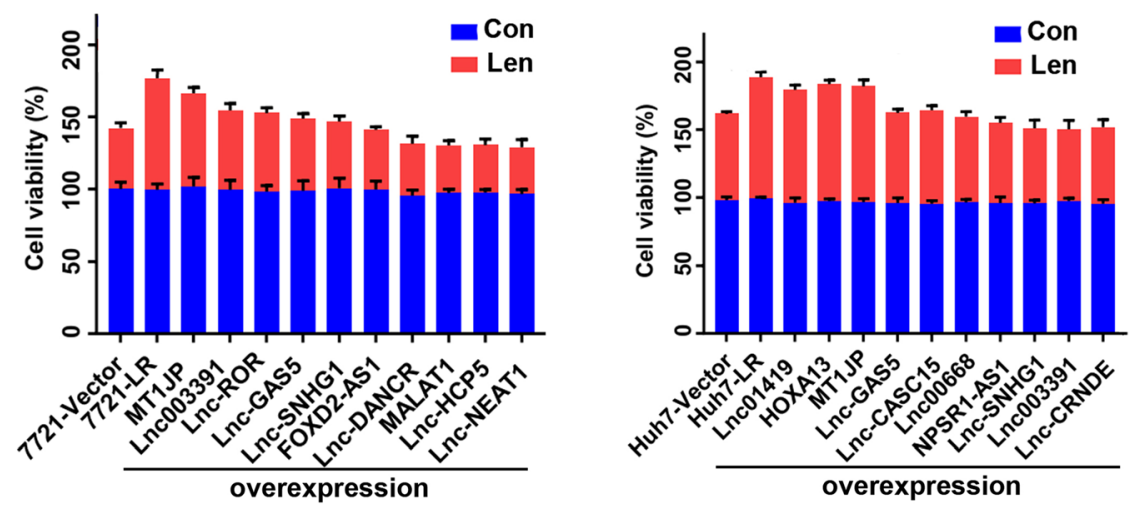

d

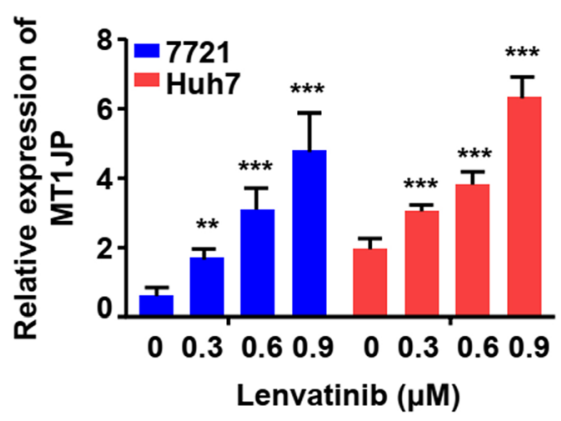

e

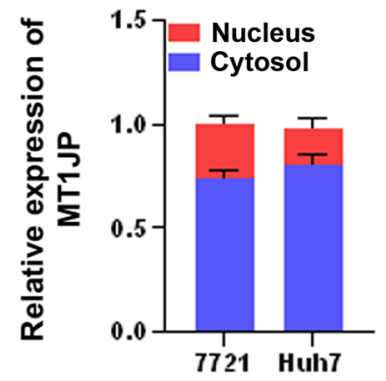

\subsection{MT1JP contributes to Lenvatinib resistance in HCC cells by inactivating the apoptosis pathway}

We next examined the relationship between MT1JP expression and Lenvatinib resistance. To this end, mock (siCon) and anti-MT1JP (siMT1JP) siRNA transfected LR-HCC cells were incubated with Lenvatinib for $48 \mathrm{~h}$. The efficiency of siRNA-mediated silencing was confirmed by qRT-PCR (Fig. 3a). Next, we found that in LR-HCC cells, MT1JP silencing resulted in alterations in cell viability (Fig. 3b), leading to a 2.2-fold upregulation of the apoptotic rate (Fig. 3c-d) and an induction of the apoptosis signaling pathway, after Lenvatinib exposure (Fig. 3e). The anti-apoptotic abilities of LR-HCC cells were found to be decreased after MT1JP
Fig. 3 MT1JP confers poor Lenvatinib response on HCC cells through inhibiting apoptosis. a. LR-7721, LR-Huh7 and parental HCC cells were transfected with either control vector (siCon) or two siRNAs targeting MT1JP (siMT1JP-1 and siMT1JP-2) and incubated for $48 \mathrm{~h}$ in the absence or presence of Lenvatinib $(0.5 \mu \mathrm{M})(\mathrm{Con}$ and Len). The transfection efficiencies were confirmed by qRT-PCR. b. Viabilities (\%) of the above cells (a) treated with or without Lenvatinib (Len and Con) were calculated $(n=3)$. c. Representative images taken from the above cells (a) incubated with or without Lenvatinib and stained by TUNEL (red) and DAPI (blue) (Scale bars: $10 \mu \mathrm{m}$ ). d. Quantification of TUNEL positive cells in (c). e. Lysates from the above cells (a) incubated with or without Lenvatinib were subjected to Western blot analysis. Band densities were normalized to GADPH. Compared using Student's t test, $* p<0.05, * * p<0.01, * * * p<0.001$ 


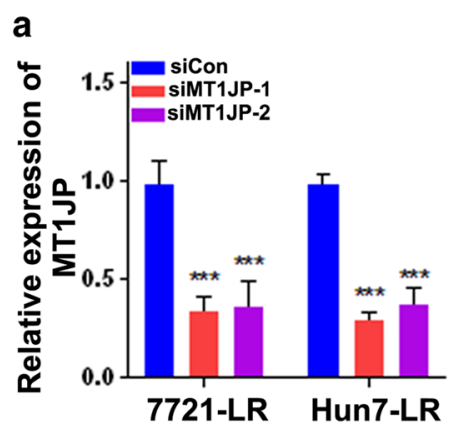

b
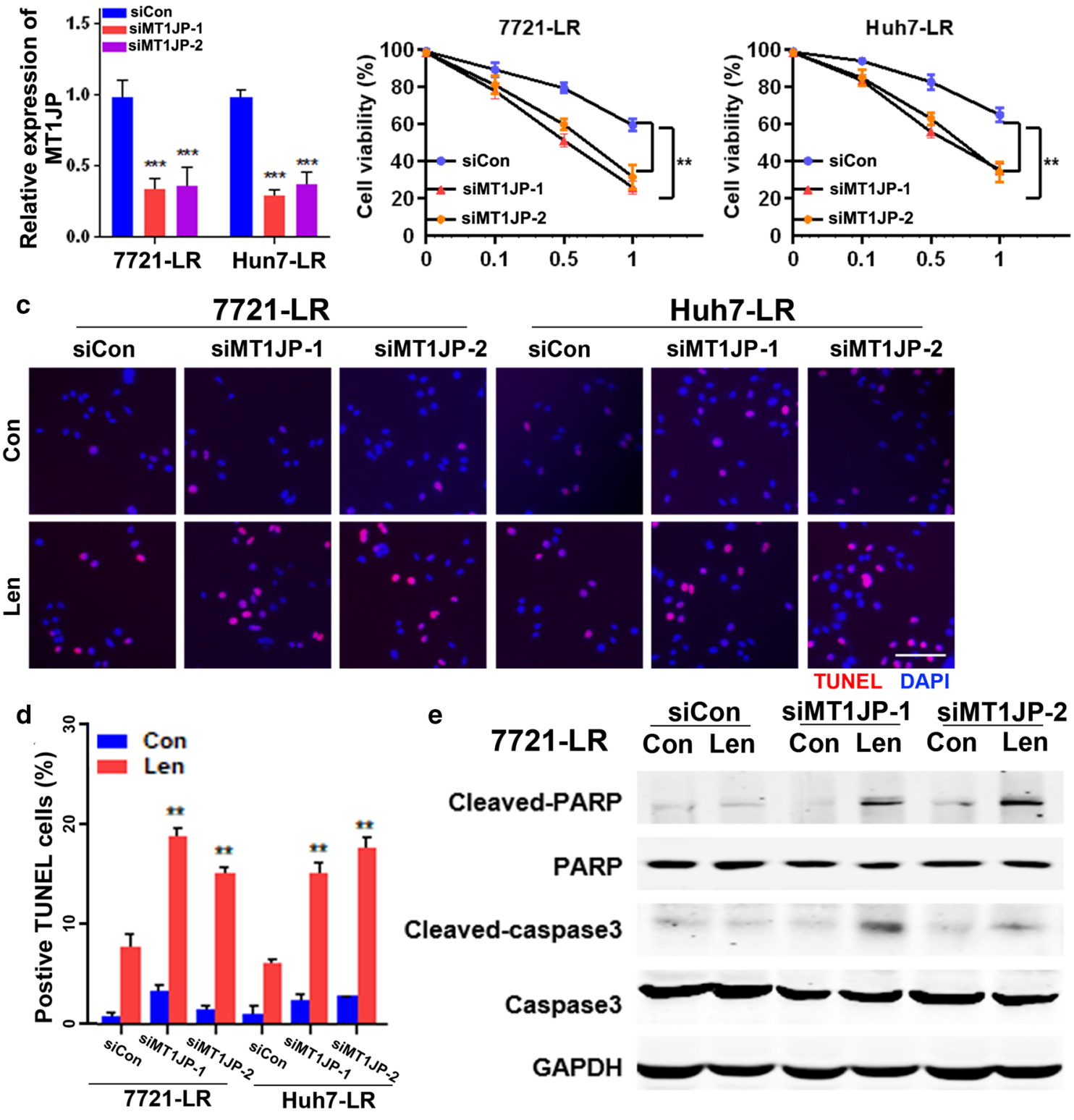

e

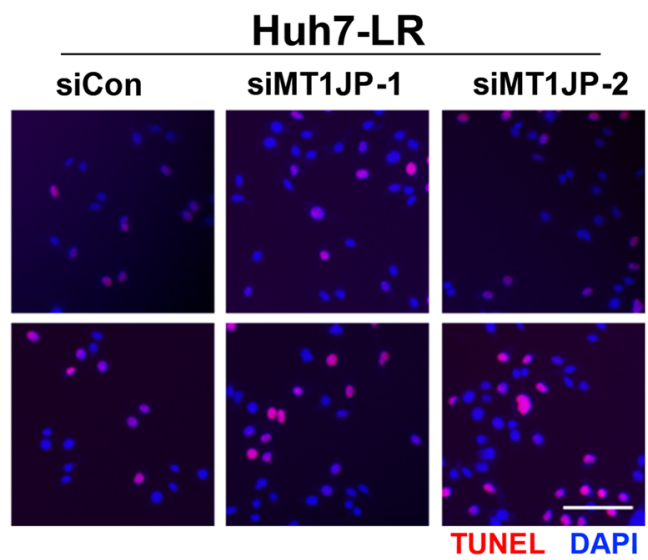

7721-LR Con Len $\frac{\text { Con Len }}{\text { Con Len }}$

Cleaved-PARP

PARP

Cleaved-caspase 3

Caspase3

GAPDH

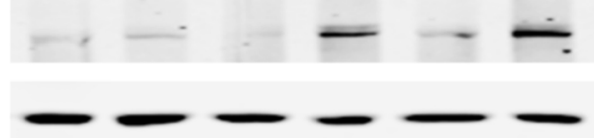

siCon siMT1JP-1 siMT1JP-2
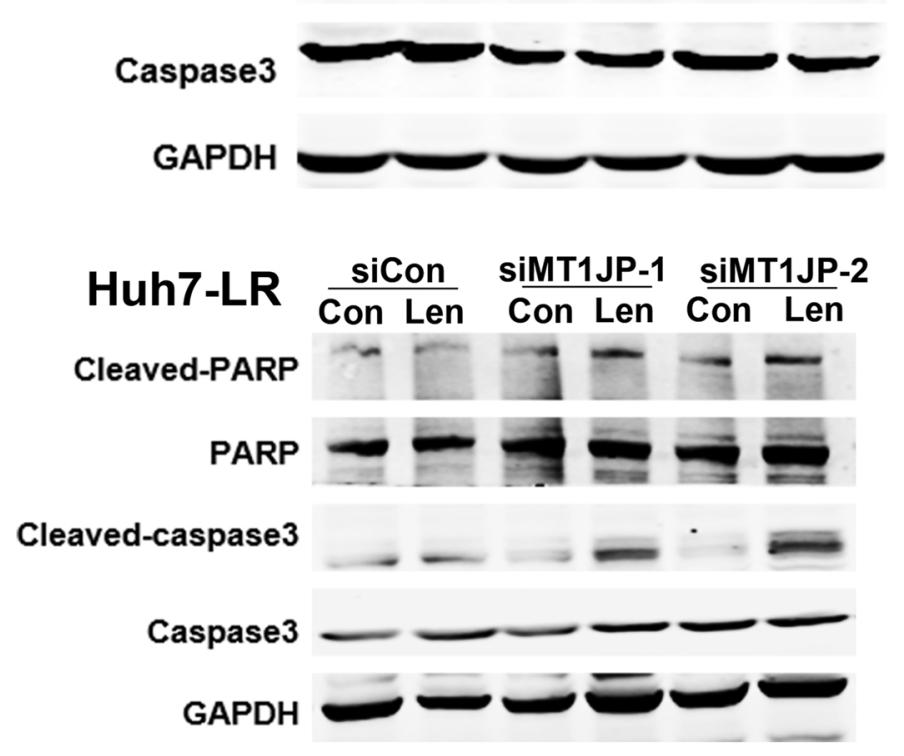

silencing, which was confirmed by expression analysis of key apoptotic proteins (Fig. 3e). Both MT1JP overexpressing SMMC-7721 and Huh7 cells acquired resistance to
Lenvatinib (Fig. S3A-B). Concordantly, we found that exogenous MT1JP overexpression (MT1JP) decreased the apoptotic rates by 2.1- and 2.4-fold compared to the mock group 
(vector) (Fig. S3C). MT1JP overexpression also led to downregulation of the expression of cleaved-Caspase-3 and PARP (Fig. S3D). Interestingly, overexpression of MT1JP did not significantly change HCC cell migration after Lenvatinib treatment (Fig. S3E).

\subsection{MT1JP serves as a ceRNA for miR-24-3p to regu- late HCC cell apoptosis}

Previously, it has been reported that LncRNAs can regulate expression levels and biological functions of specific miRNAs, either acting as molecular sponges or as competing endogenous RNAs (ceRNAs) [18]. In order to identify possible targets of MT1JP, we used miRDB (http://mirdb.org/ miRDB/custom.html), AnnoLnc (http://annolnc.cbi.pku.edu. cn) and RAID (http://www.rna-society.org/raid/) online prediction tools to find candidate miRNAs that may be regulated by MT1JP (Fig. 4a). Of the miRNAs that fit the criteria, only miR-24-3p emerged as a candidate, as its predicted binding sites were shared by MT1JP (Fig. S4A). Next, we assessed the expression of miR-24-3p in control and LR cells and found that its expression was lower in cell lines in which the MT1JP mRNA levels were relatively higher (Fig. 4b). For a further confirmation of a putative interaction between MT1JP and miR-24-3p, we subcloned MT1JP (wild type [WT] and mutant [Mut]) downstream of the firefly luciferase gene into a pmirGLO vector, based on online RAID analysis (Fig. 4c), and next performed a luciferase reporter assay. We found that anti-miR-24-3p markedly increased the luciferase activity of the WT-MT1JP construct, but not of the Mut-MT1JP construct (Fig. 4d). These results indicate that miR-24-3p can bind directly to MT1JP at its miRNA recognition site.

We next explored the effect of miR-24-3p expression on apoptosis and Lenvatinib sensitivity of HCC cells. To this end, we transfected miR-NC/miR-24-3p inhibitor into SMMC-7721 or Huh-7 cells and miR-NC/miR-24-3p mimics into LR-HCC cells. The respective expression effects were confirmed by qRT-PCR (Fig. S4B). Since miR-24-3 inhibition did not affect the expression of MT1JP (Fig. S4C), we first analyzed the relation between cell viability in response to Lenvatinib exposure and relative miR-24-3p expression in the HCC cells and found that miR-24-3p overexpression decreased the viability of Lenvatinib treated LR-HCC cells (Fig. S4D). Opposite effects were observed in HCC cells after inhibition of miR-24-3p (Fig. S4E). Subsequently, we investigated the apoptotic rates after transfection. Using TUNEL and Western blotting assays, we found that miR-24-3p had a negative effect on the apoptosis pathway (Fig. 4e-g and Fig. S4F). Taken together, these data indicate that MT1JP serves as a ceRNA for miR-24-3p to regulate the apoptosis pathway in HCC cells.

\section{5 miR-24-3p regulates HCC cell apoptosis suppression through BCL2L2}

To assess the role of miR-24-3p in the apoptosis regulatory loop, online prediction tools were used to identify candidate targets. We identified 171 genes as candidates (Fig. 5a). Further GO analysis revealed that 4 of the 171 genes were associated with the apoptosis pathway (Fig. 5b). The expression of all four genes tended to be increased in miR-24-3p silenced HCC cells (Fig. S5A), but only cells overexpressing Bcl-2 like 2 (BCL2L2) exhibited a better cell viability after exposure to Lenvatinib compared to the other three genes (Fig. S5B-C). Moreover, the expression of BCL2L2 was dramatically increased after MT1JP overexpression, which could be reversed after miR-24-3p mimics transfection (Fig. 5c). Conversely, we found that overexpression of miR-24-3p in LR-HCC cells led to a decreased expression of BCL2L2, which could be reversed by transfection of a wild type MT1JP vector but not of a mutant MT1JP vector (Fig. S5D), which is in accordance with previous results. To better understand the role of BCL2L2 in LR formation, BCL2L2 was overexpressed in SMMC-7721 and Huh7 cells and silenced in LR-7721 and Huh7 (siBCL2L2) cells. The respective transfection efficiencies were confirmed (Fig. S5E). We first analyzed the correlation between $\mathrm{IC}_{50}$ in response to Lenvatinib and BCL2L2 expression in the transfected LR-HCC cells and found that these cells became sensitive to Lenvatinib treatment after inhibition of BCL2L2 expression (Fig. 5d-e). Compared to the control group, BCL2L2 silencing increased the sensitivity of HCC cells to Lenvatinib via apoptosis induction, whereas exogenous overexpression of BCL2L2 in LRHCC cells decreased the levels of cleaved-Caspase 3 and PARP (Fig. 5f-h). Concordant results were obtained with HCC cells overexpressing BCL2L2 (Fig. 5d-e and S5F-H). These data suggest that miR-24-3p-mediated BCL2L2 suppression is involved in MT1JP-induced poor Lenvatinib sensitivity.

\subsection{The MT1JP/miR-24-3p axis contributes to in vivo Lenvatinib resistance in HCC PDX models}

We processed 26 fresh HCC specimens from different patients and successfully established 17 PDX models. The xenografted NSG mice orally received Lenvatinib $(50 \mathrm{mg} / \mathrm{kg})$ once the PDX tumors reached about $1.0 \mathrm{~cm}$ in diameter. The percentage of tumor regression was calculated after continuous administration of Lenvatinib for two weeks. A $>40 \%$ decrease in tumor volume was designated as Lenvatinib sensitive (5/17, $29.4 \%)$, and a $<20 \%$ decrease as Lenvatinib insensitive $(5 / 17$, $29.4 \%$, Fig. 6a and S6 A). The Lenvatinib sensitive group exhibited a lower expression of MT1JP and a higher expression of miR-24-3p compared to the Lenvatinib insensitive group (Fig. 6b), which is in accordance with the in vitro 
Fig. 4 MT1JP acts as a ceRNA for miR-24-3p to regulate the apoptotic signaling pathway. a. Venn diagram showing overlapping miRNAs that are predicted to bind to MT1JP. b. Expression analysis of miR-24-3p measured in Lenvatinib resistant and parental HCC cells by qRTPCR. c. Binding sequences of MT1JP and miR-24-3p based on bioinformatics analysis and binding sites of WT (WT-MT1JP) and mutant (Mut-MT1JP) regions. d. Dual-luciferase reporter assays to examine the potential interaction of MT1JP and miR-24-3 $(n=4)$. e-g. SMMC-7721 and Huh7 cells were transfected with miR-NC (NC) or miR-24-3p inhibitor (inhibitor), while LR-7721 and LRHuh7 cells were transfected with miR-NC (NC) or miR-24-3p mimics (mimics). Apoptosis through TUNEL (e-f) and Western blotting (g) assays was detected in these transfected cell lines. (Scale bars: $10 \mu \mathrm{m}$ ). Data represent three independent experiments. Compared using Student's t test, ${ }^{*} p<0.05$, $* * p<0.01, * * * p<0.001$ a

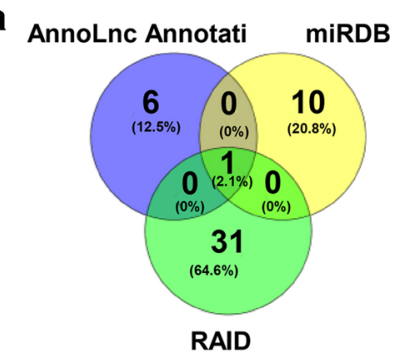

C

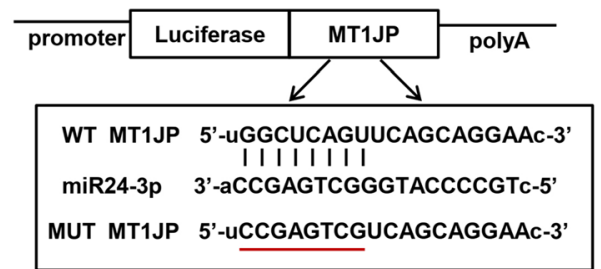

e
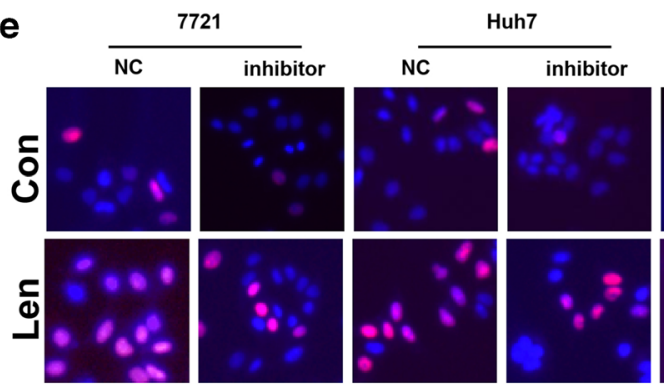

g

f

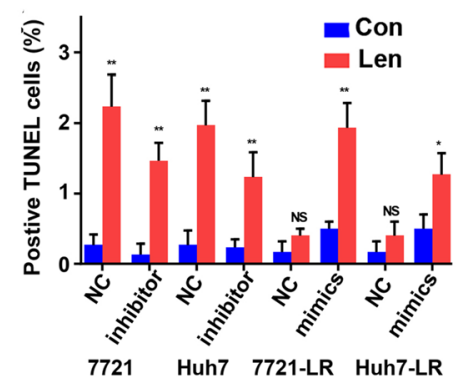

Cleaved-caspase3 b

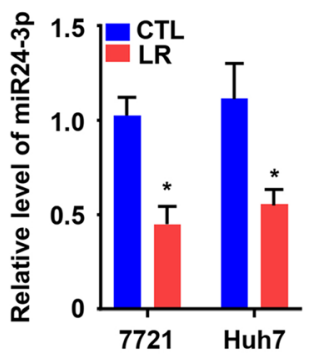

d

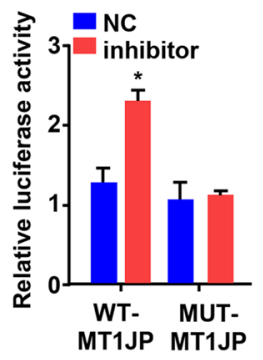

$$
\text { 7721-LR }
$$
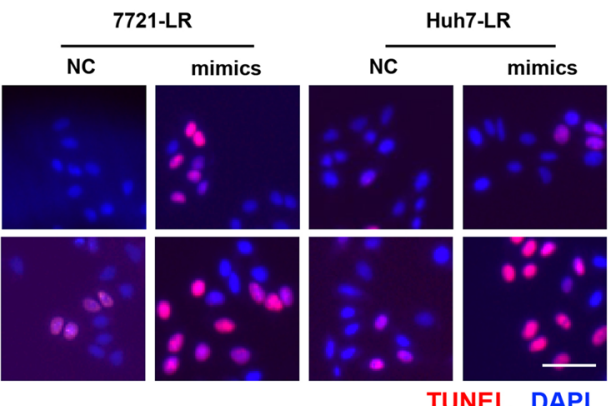

TUNEL DAPI
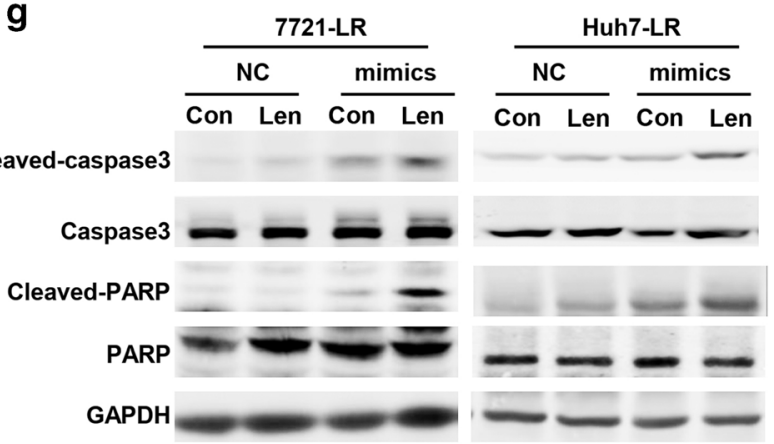

HCC cell line data. Moreover, Western blotting and IHC evaluation of the apoptosis pathway indicated that in the Lenvatinib sensitive group the apoptosis signals were induced to promote tumor degradation (Fig. 6c-e). These results further confirm our in vitro findings in HCC cell lines.

\subsection{MT1JP and BCL2L2 overexpression attenuate in vivo $\mathrm{HCC}$ sensitivity to Lenvatinib}

To verify the effects of MT1JP and BCL2L2 on sensitivity to Lenvatinib in vivo, we employed a xenograft model with SMMC-7721 cells. After the volume of subcutaneous tumors reached $\sim 50 \mathrm{~mm}^{3}$, different doses of Lenvatinib were orally administered after which the tumor volumes were assessed every 5 days. Considering the size of the tumors, a dose of $50 \mathrm{mg} / \mathrm{kg}$ was found to be appropriate for the mice (Fig. S7A-F). In addition, SMMC-7721 cells with stable MT1JP overexpression or BCL2L2 inhibition and SMMC-7721 cells transfected with an empty vector or inhibition control as negative controls were injected into mice, which were not treated with $50 \mathrm{mg} / \mathrm{kg}$ Lenvatinib until the tumors reached $\sim 50 \mathrm{~mm}^{3}$. Consistent with our in vitro observations, we found that tumors overexpressing MT1JP or with BCL2L2 inhibition grew faster than those derived from control cells (Fig. 7a-b). 
Fig. 5 BCL2L2 suppression, mediated by miR-24-3p, is involved in MT1JP-mediated Lenvatinib tolerance. a. Venn diagram showing overlapping genes that are predicted to bind to miR-24-3p. b. GO enrichment graph of the common 171 genes predicted in (a). $P$ values (white) and different gene numbers (black) are listed in the graph. c. HCC cell lines were transfected with either MT1JP vector or miR24-3p mimics. Expression of BCL2L2 was detected by Western blotting. d-e. SMMC7721 and Huh7 cells were transfected with control vector (vector) or BCL2L2, while LR7721 and LR-Huh7 cells were transfected with control siRNA (siNC) or BCL2L2 siRNA (siBCL2L2). The viability (\%) of these transfected cells incubated with Lenvatinib $(5 \mu \mathrm{M})$ was calculated. f-h. Apoptosis through TUNEL (f-g) and Western blotting (h) assays was detected in the above cells (e). Data represent three independent experiments. (Scale bars: $10 \mu \mathrm{m}$ ). Compared using Student's t test, $* p<0.05$, $* * p<0.01, * * * p<0.001$ a

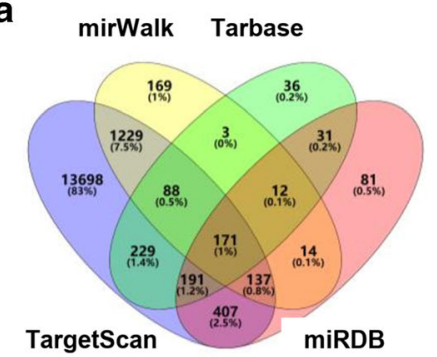

C

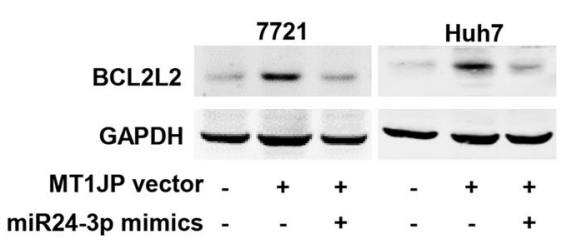

b

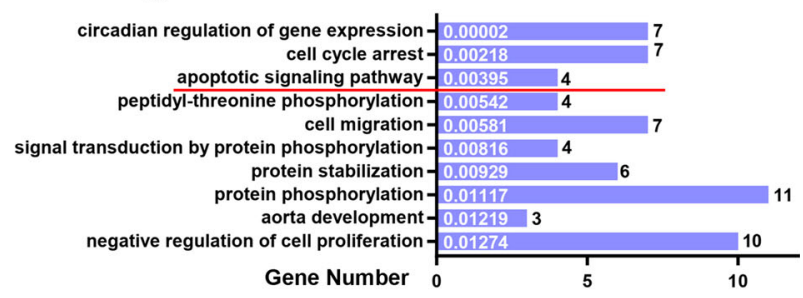

d

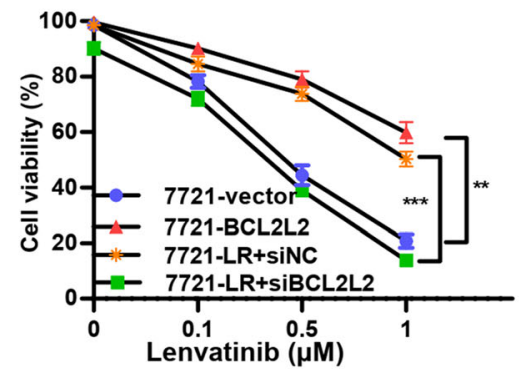

e
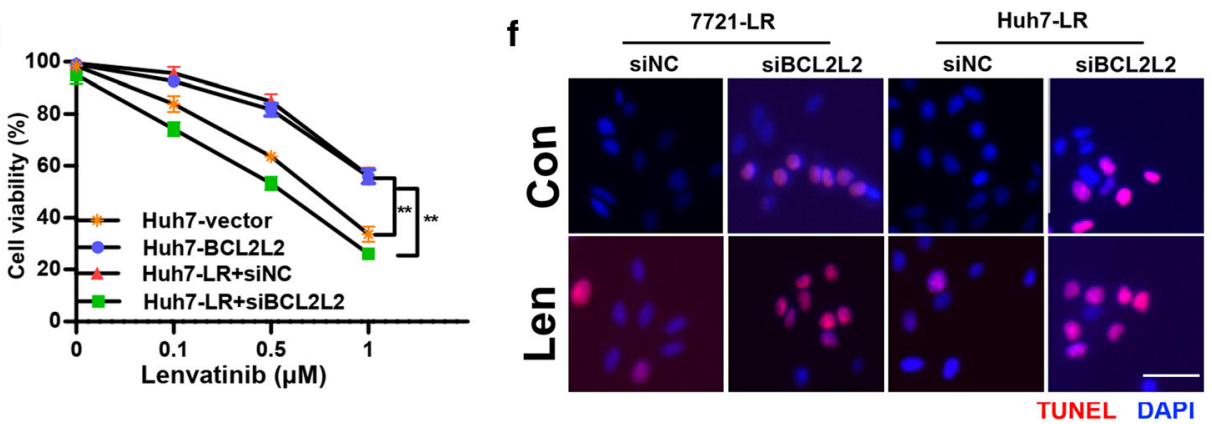

g

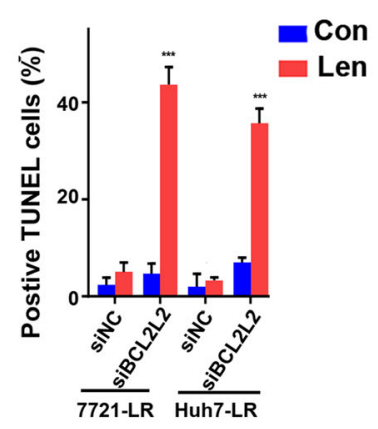

h

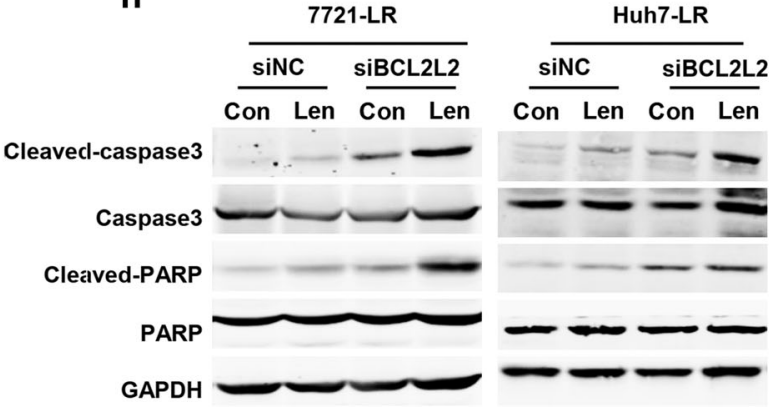

We also found no significant alternations after synchronous MT1JP overexpression and BCL2L2 inhibition compared to the control group (Fig. 7a-b). These results further suggest that MT1JP can promote Lenvatinib resistance via the miR-24-3p/ BCL2L2 axis. In addition, we applied Western blotting to assess apoptosis pathway alterations in the tumors. We found that tumors derived from stably MT1JP- or BCL2L2transfected SMMC-7721 cells presented with a significantly reduced apoptosis compared with tumors derived from control cells, and that this effect was reversed by synchronous MT1JP overexpression and BCL2L2 inhibition (Fig. 7c). Using IHC, similar results were obtained (Fig. 7d). Therefore, we conclude that our in vivo experiments underscore the results of the in vitro experiments.

\section{Discussion}

Lenvatinib is a novel TKI drug for advanced HCC and is, next to Sorafenib, approved by many international and national authorities. The survival benefit from Lenvatinib may, however, be hampered by therapy resistance as is true for almost all TKIs [19]. Therefore, it is essential to explore the mechanisms underlying Lenvatinib resistance in HCC. In the present study, we found that Lenvatinib-induced upregulation of LncRNA MT1JP (MT1JP) contributes to Lenvatinib resistance (LR) by inhibiting activation of the apoptosis pathway via the miR-24-3p/BCL2L2 axis. MT1JP silencing decreased the effect of Lenvatinib against LR-HCC cells that were refractory to Lenvatinib-induced proliferation inhibition and 


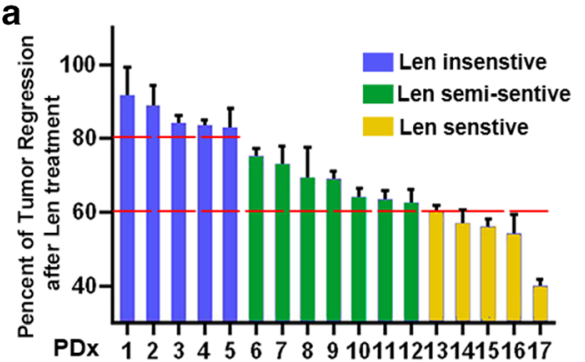

C

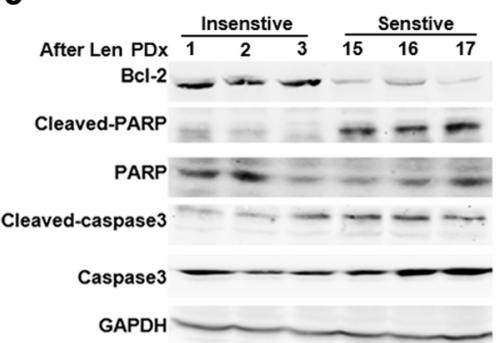

e

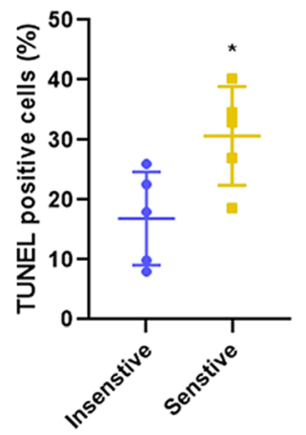

Fig. $6 \mathrm{MT1JP} / \mathrm{miR}-24-3 \mathrm{p}$ axis contributes to Lenvatinib resistance in a HCC PDX model. a. Seventeen fragments of PDX tumors from HCC patients were used for Lenvatinib sensitivity screening. NSG mice received oral Lenvatinib administration $(50 \mathrm{mg} / \mathrm{kg}$ ) once the founder PDX tumors reached $\sim 1.0 \mathrm{~cm}$ in diameter. The percentage of tumor regression was calculated as reduced volume after Lenvatinib treatment, normalized to the primary tumor volume. A $>40 \%$ decrease in tumor volume was termed as Lenvatinib sensitive (Len sensitive, 5/17, 29.4\%), and $\mathrm{a}<20 \%$ decrease as Lenvatinib insensitive (Len insensitive, 5/17,

apoptosis in vitro and in vivo. We showed that Lenvatinib promoted the apoptosis pathway in a concentrationdependent manner and that LR increased the anti-apoptotic effects of Lenvatinib in both SMMC-7721 and Huh7 cells, which implies that LR formation is probably associated with inhibition of the apoptosis pathway. Several other studies have shown that the apoptosis pathway may be activated after Lenvatinib stimulation [20-22]. Although some studies reported that Lenvatinib did not dose-dependently suppress tumor growth and apoptosis in cell lines [14], it should be noted that instant culturing with Lenvatinib may not be enough to affect the apoptosis pathway [20]. We found that Lenvatinibtreated cells showed a marked suppression of viability and upregulation of apoptosis signaling, similar to previous b
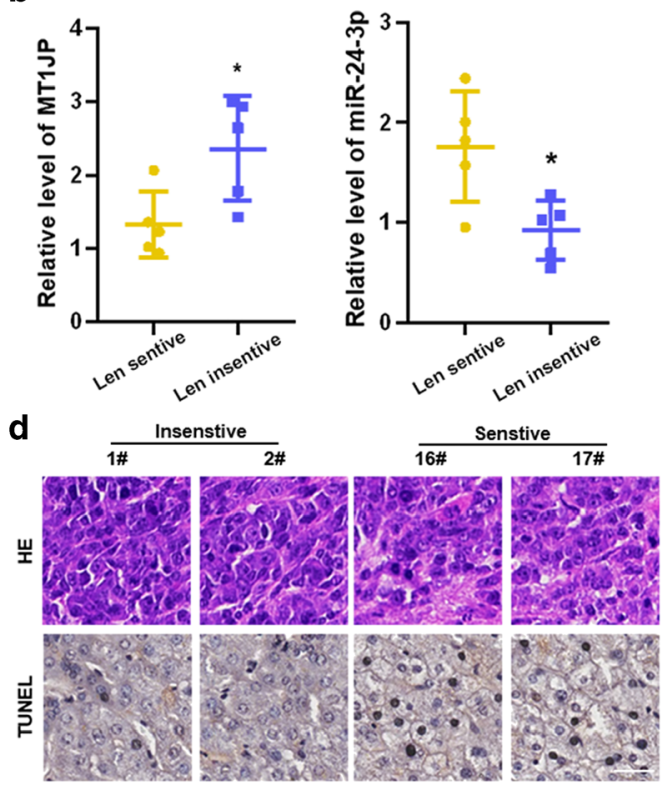

$29.4 \%$ ). b. Relative expression levels of MT1JP and miR-24-3p in Len sensitive and insensitive groups measured by qRT-PCR. c. Lysates from Len sensitive and insensitive PDX cases after Lenvatinib treatment were subjected to Western blot analysis. Band densities were normalized to GADPH. d-e. Representative images of H\&E staining and TUNEL staining are shown. (Scale bars: $50 \mu \mathrm{m}$ ) Quantification of TUNEL positive cells in (c) is shown in (e). Compared using Student's t test, ${ }^{*} p<0.05$, $* * p<0.01, * * * p<0.001$

studies $[20,21]$. This effect could be reversed when the HCC cells acquired resistance to Lenvatinib.

The proposed mechanisms by which MT1JP activates the anti-apoptosis pathway and its regulation by miR-24-3p in LR-HCC cells are depicted schematically in Fig. 7e. MT1JP has previously been reported to act as a tumor suppressor in breast and bladder cancer $[23,24]$ and it has been reported that MT1JP may inhibit the proliferation, invasion and migration of tumor cells [25, 26]. Zhu et al. reported that MT1JP could inhibit the tumorigenesis and enhance the cisplatin sensitivity of breast cancer cells through competitively binding to miR24-3p [22]. In this study, overexpression of MT1JP in breast cancer cells significantly inhibited their proliferation and enhanced their cisplatin sensitivity, which seems to be 

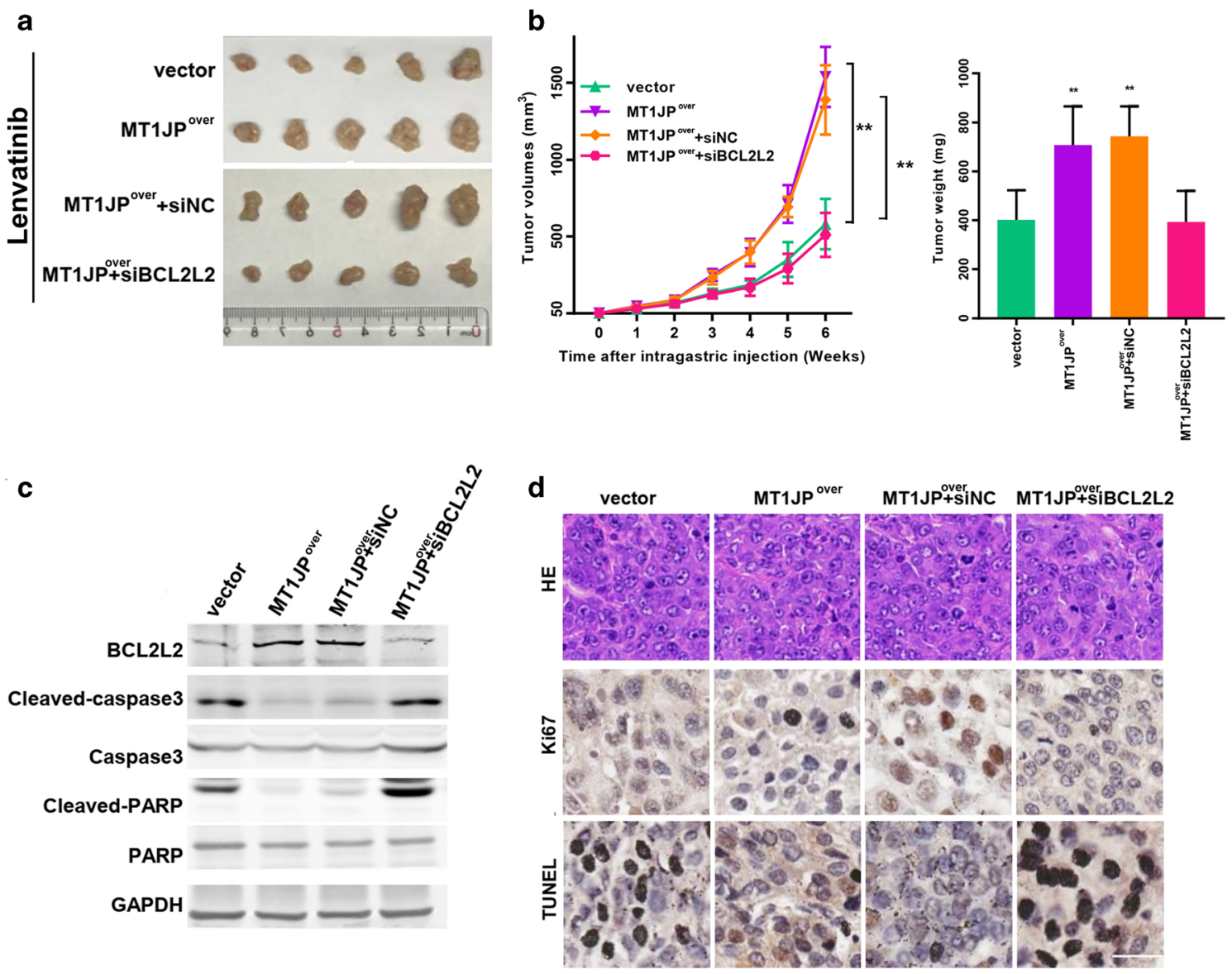

e

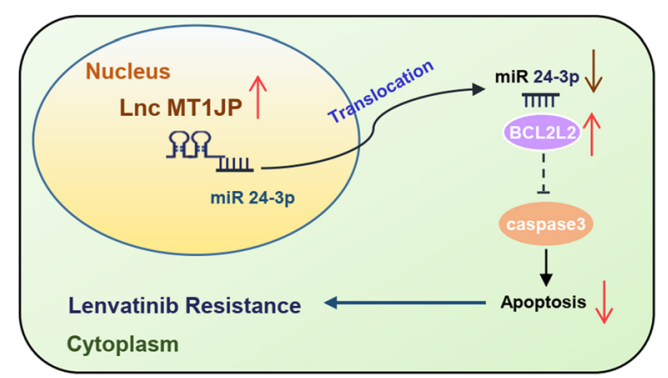

Fig. 7 MT1JP or BCL2L2 overexpression attenuate sensitivity to Lenvatinib in vivo. a. MT1JP (MTJ1 $\mathrm{P}^{\text {over }}$ ) or control vector (vector) were overexpressed in SMMC-7721 cells followed by interfering with BCL2L2 (MT1JP ${ }^{\text {over }}+$ siBCL2L2) or its control $\left(\mathrm{MT}^{\mathrm{J}} \mathrm{JP}^{\text {over }}+\mathrm{siNC}\right)$. These cells were implanted into nude mice. When the average tumor volume reached $\sim 50 \mathrm{~mm}^{3}$, the mice were randomly subdivided into four groups and administered $50 \mathrm{mg} / \mathrm{kg}$ Lenvatinib daily. Tumor sizes were

calculated every 5 days. Images of subcutaneous xenograft tumors were taken. b. Growth curves (left) or tumor burden (right) of the subcutaneous xenograft tumors were calculated ( $n=12$ mice for each group). c. Lysates from the above tumors (a) were subjected to Western blot analysis. d. Representative images of H\&E staining, immunohistochemical staining (for Ki67) and TUNEL staining are shown. (Scale bars: $50 \mu \mathrm{m}$ ). e. Schematic overview of MT1JP and apoptosis regulatory signaling

discordant with our results. It should be noted, however, that breast cancer cells are sensitive to cisplatin when proliferating, and that blocking the cell cycle will affect the efficacy of cisplatin. In contrast, $\mathrm{HCC}$ cells are quite insensitive to chemotherapy and Lenvatinib inhibits HCC proliferation through the apoptosis signaling pathway, which may explain the differences in results between breast cancer and HCC cells.
It is well-known that cross-talk between Lnc-RNAs and miRNAs is involved in many biological processes [27]. Therefore, in the present study, we further explored the interaction between MT1JP and miR-24-3p in the apoptosis pathway. Previously, miR-24-3p has been shown to play an important role in regulating cell growth and metastasis in various types of cancer [22, 28, 29]. MiR-24-3p has also been identified as a key miRNA associated with activation of the 
apoptosis pathway [29]. Intriguingly, our study revealed a novel regulatory pathway, in which Lenvatinib induces expression of MT1JP, acting as a competing endogenous RNA with miR-24-3p, regulating the anti-apoptosis protein BCL2L2. BCL2L2 is a member of the Bcl-2 protein family, which act as anti-apoptotic regulators and reduce apoptosis under cytotoxic conditions [30-32]. Our results indicate that high expression of BCL2L2 leads to resistance to Lenvatinib and that downregulation of this protein may increase HCC sensitivity to Lenvatinib in vivo and in vitro, which agrees with a previous study showing that Bcl-2 can effectively curb ovarian cancer cell apoptosis through resistance to cisplatin [33]. This notion may point at a promising therapeutic target for the treatment of Lenvatinib-resistant HCC.

One caveat of the present study is that it does not address the mechanism of invasion of LR HCC cells and the possible role of the MT1JP/miR-24-3p/BCL2L2 axis therein. In addition, the results obtained in the cell and animal models have not been validated in primary tumor tissues collected from HCC patients who have developed Lenvatinib resistance. Thus, validation in clinical LR-HCC tissues needs to be performed in future studies.

\section{Conclusions}

In the present study we show that Lnc-RNA MT1JP contributes to HCC Lenvatinib resistance by inhibiting apoptosis through regulating the miR-24-3p/BCL2L2 axis. MT1JP silencing enhanced the efficacy of Lenvatinib in suppressing LR-HCC cell propagation by promoting apoptosis in vitro in cell models and in vivo in animal models. MiR-24-3p was able to suppress the expression of BCL2L2, which is an anti-apoptotic regulator, and inhibited $\mathrm{HCC}$ cell resistance to Lenvatinib treatment. Our results suggest that MT1JP or BCL2L2 may serve as targets for overcoming Lenvatinib resistance in HCC.

\footnotetext{
Abbreviations BCL2L2, Bcl-2 Like 2; ceRNA, competing endogenous RNA; FGF, fibroblast growth factor; GAPDH, glyceraldehyde-3-phosphate dehydrogenase; $\mathrm{HBV}$, hepatitis $\mathrm{B}$ virus; $\mathrm{HCV}$, hepatitis $\mathrm{C}$ virus; HCC, hepatocellular carcinoma; LR, Lenvatinib resistance; MT1JP, LncMT1JP; PDGF, platelet derived growth factor; PARP, poly ADP-ribose polymerase; RNA-seq, RNA sequencing; RNA-seq, RNA sequencing; RT-PCR, real time-polymerase chain reaction; TKI, tyrosine kinase inhibitor; TUNEL, TdT-mediated dUTP Nick-End Labeling; WB, western blot; VEGF, vascular endothelial growth factor.
}

Supplementary Information The online version contains supplementary material available at https://doi.org/10.1007/s13402-021-00605-0.

Acknowledgments We thank Jihui Zhu for technical assistance.

Author contributions Yu Ting: acquisition of data, analysis and interpretation of data, and statistical analysis; Jiajian Yu: analysis and interpretation of data and drafting of the manuscript; $\mathrm{Lu} \mathrm{Lu}$ and Yize Zhang: acquisition of data; Yadong Zhou, Fengling Huang, Lu Sun, Yong Zhou, and Zhixian Guo: manuscript editing; Guojun Hou: constructed of PDX models, obtained study funding, and study supervision; Zihui Dong: study concept and design, analysis and interpretation of data, drafting of the manuscript, critical revision of the manuscript for important intellectual content, obtained study funding, and study supervision; Bibo Wang: study concept and design, analysis and interpretation of data, critical revision of the manuscript for important intellectual content, obtained study funding, and study supervision. All authors have read and approved the manuscript for publication.

Funding This research was supported by projects from the National Natural Science Foundation of China $(81902502,81802983)$ and the National Natural Science Foundation of Chongqing (csct2019jcyjmsxmX0659).

Data availability The data used and analyzed during the current study are available from the corresponding author upon reasonable request.

\section{Declarations}

Ethics approval and consent to participate All procedures in this study were performed in accordance with the ethical standards of the Animal Care and Use Committee of Nanjing University.

Consent for publication Not applicable.

Competing interests The authors declare that they have no competing interests.

Open Access This article is licensed under a Creative Commons Attribution 4.0 International License, which permits use, sharing, adaptation, distribution and reproduction in any medium or format, as long as you give appropriate credit to the original author(s) and the source, provide a link to the Creative Commons licence, and indicate if changes were made. The images or other third party material in this article are included in the article's Creative Commons licence, unless indicated otherwise in a credit line to the material. If material is not included in the article's Creative Commons licence and your intended use is not permitted by statutory regulation or exceeds the permitted use, you will need to obtain permission directly from the copyright holder. To view a copy of this licence, visit http://creativecommons.org/licenses/by/4.0/.

\section{References}

1. Z.G. Han, Functional genomic studies: insights into the pathogenesis of liver cancer. Annu Rev Genomics Hum Genet 13, 171-205 (2012)

2. H.B. El-Serag, K.L. Rudolph, Hepatocellular carcinoma: epidemiology and molecular carcinogenesis. Gastroenterology 132, 2557 2576 (2007)

3. R.L. Siegel, K.D. Miller, A. Jemal, Cancer statistics, 2016. CA Cancer J Clin 66, 7-30 (2016)

4. P.A. Farazi, R.A. DePinho, Hepatocellular carcinoma pathogenesis: from genes to environment. Nat Rev Cancer 6, 674-687 (2006)

5. J. Bruix, L.G. da Fonseca, M. Reig, Insights into the success and failure of systemic therapy for hepatocellular carcinoma. Nat Rev Gastroenterol Hepatol 16, 617-630 (2019)

6. J. Bruix, S. Qin, P. Merle, A. Granito, Y.H. Huang, G. Bodoky, M. Pracht, O. Yokosuka, O. Rosmorduc, V. Breder, R. Gerolami, G. 
Masi, P.J. Ross, T. Song, J.P. Bronowicki, I. Ollivier-Hourmand, M. Kudo, A.L. Cheng, J.M. Llovet, R.S. Finn, M.A. LeBerre, A. Baumhauer, G. Meinhardt, G. Han, R. Investigators, Regorafenib for patients with hepatocellular carcinoma who progressed on sorafenib treatment (RESORCE): A randomised, double-blind, placebo-controlled, phase 3 trial. Lancet 389, 56-66 (2017)

7. A.B. El-Khoueiry, B. Sangro, T. Yau, T.S. Crocenzi, M. Kudo, C. Hsu, T.Y. Kim, S.P. Choo, J. Trojan, T.H.R. Welling, T. Meyer, Y.K. Kang, W. Yeo, A. Chopra, J. Anderson, C. Dela Cruz, L. Lang, J. Neely, H. Tang, H.B. Dastani, I. Melero, Nivolumab in patients with advanced hepatocellular carcinoma (CheckMate 040): An open-label, non-comparative, phase 1/2 dose escalation and expansion trial. Lancet 389, 2492-2502 (2017)

8. M. Kudo, R.S. Finn, S. Qin, K.H. Han, K. Ikeda, F. Piscaglia, A. Baron, J.W. Park, G. Han, J. Jassem, J.F. Blanc, A. Vogel, D. Komov, T.R.J. Evans, C. Lopez, C. Dutcus, M. Guo, K. Saito, S. Kraljevic, T. Tamai, M. Ren, A.L. Cheng, Lenvatinib versus sorafenib in first-line treatment of patients with unresectable hepatocellular carcinoma: A randomised phase 3 non-inferiority trial. Lancet 391, 1163-1173 (2018)

9. J. Matsui, Y. Yamamoto, Y. Funahashi, A. Tsuruoka, T. Watanabe, T. Wakabayashi, T. Uenaka, M. Asada, E7080, a novel inhibitor that targets multiple kinases, has potent antitumor activities against stem cell factor producing human small cell lung cancer H146, based on angiogenesis inhibition. Int J Cancer 122, 664-671 (2008)

10. O. Tohyama, J. Matsui, K. Kodama, N. Hata-Sugi, T. Kimura, K. Okamoto, Y. Minoshima, M. Iwata, Y. Funahashi, Antitumor activity of lenvatinib (e7080): an angiogenesis inhibitor that targets multiple receptor tyrosine kinases in preclinical human thyroid cancer models. J Thyroid Res 2014, 638747 (2014)

11. L. Rimassa,M.A. Worns, Navigating the new landscape of secondline treatment in advanced hepatocellular carcinoma. Liver Int 40, 1800-1811 (2020)

12. L. Hamieh, R.L. Beck, V.H. Le, J.J. Hsieh, The efficacy of Lenvatinib plus Everolimus in patients with metastatic renal cell carcinoma exhibiting primary resistance to front-line targeted therapy or immunotherapy. Clin Genitourin Cancer 18, 252-257.e2 (2020)

13. R. Yu, M. Wang, X. Zhu, Z. Sun, A. Jiang, H. Yao, Therapeutic effects of lenvatinib in combination with rAd-p 53 for the treatment of non-small cell lung cancer. Oncol Lett 16, 6573-6581 (2018)

14. S. Ogasawara, Y. Mihara, R. Kondo, H. Kusano, J. Akiba, H. Yano, Antiproliferative effect of Lenvatinib on human liver cancer cell lines in vitro and in vivo. Anticancer Res 39, 5973-5982 (2019)

15. S.M. Ferrari, G. Bocci, T. Di Desidero, G. Elia, I. Ruffilli, F. Ragusa, P. Orlandi, S.R. Paparo, A. Patrizio, S. Piaggi, C. La Motta, S. Ulisse, E. Baldini, G. Materazzi, P. Miccoli, A. Antonelli, P. Fallahi, Lenvatinib exhibits antineoplastic activity in anaplastic thyroid cancer in vitro and in vivo. Oncol Rep 39, 2225$2234(2018)$

16. G. Wang, J. Zhuang, J. Ni, Y. Ye, S. He, W. Xia, Combined effects of Lenvatinib and iodine-131 on cell apoptosis in nasopharyngeal carcinoma through inducing endoplasmic reticulum stress. Exp Ther Med 16, 3325-3332 (2018)

17. Y. Niu, S. Altuwaijri, S. Yeh, K.P. Lai, S. Yu, K.H. Chuang, S.P. Huang, H. Lardy, C. Chang, Targeting the stromal androgen receptor in primary prostate tumors at earlier stages. Proc Natl Acad Sci U S A 105, 12188-12193 (2008)

18. J. Wang, X. Liu, H. Wu, P. Ni, Z. Gu, Y. Qiao, N. Chen, F. Sun, Q. Fan, CREB up-regulates long non-coding RNA, HULC expression through interaction with microRNA-372 in liver cancer. Nucleic Acids Res 38, 5366-5383 (2010)
19. R. Fu, S. Jiang, J. Li, H. Chen, X. Zhang, Activation of the HGF/cMET axis promotes lenvatinib resistance in hepatocellular carcinoma cells with high c-MET expression. Med Oncol 37, 24 (2020)

20. X. Ma, Y. Qiu, Y. Sun, L. Zhu, Y. Zhao, T. Li, Y. Lin, D. Ma, Z. Qin, C. Sun, L. Han, NOD2 inhibits tumorigenesis and increases chemosensitivity of hepatocellular carcinoma by targeting AMPK pathway. Cell Death Dis 11, 174 (2020)

21. S.M. Ferrari, C. La Motta, G. Elia, F. Ragusa, I. Ruffilli, L. Quattrini, S.R. Paparo, S. Piaggi, A. Patrizio, S. Ulisse, E. Baldini, G. Materazzi, P. Fallahi, A. Antonelli, Antineoplastic effect of Lenvatinib and Vandetanib in primary anaplastic thyroid cancer cells obtained from biopsy or fine needle aspiration. Front Endocrinol (Lausanne) 9, 764 (2018)

22. S.M. Kim, S.Y. Kim, C.S. Park, H.S. Chang, K.C. Park, Impact of age-related genetic differences on the therapeutic outcome of papillary thyroid cancer. Cancers (Basel) 12, 448 (2020)

23. H. Yu, S. Wang, H. Zhu, D. Rao, LncRNA MT1JP functions as a tumor suppressor via regulating miR-214-3p expression in bladder cancer. J Cell Physiol 234, 16160-16167 (2019)

24. D. Zhu, X. Zhang, Y. Lin, S. Liang, Z. Song, C. Dong, MT1JP inhibits tumorigenesis and enhances cisplatin sensitivity of breast cancer cells through competitively binding to miR-24-3p. Am J Transl Res 11, 245-256 (2019)

25. L. Yang, G. Liu, S. Xiao, L. Wang, X. Liu, Q. Tan, Z. Li, Long noncoding MT1JP enhanced the inhibitory effects of miR-646 on FGF2 in osteosarcoma. Cancer Biother Radiopharm 35, 371-376 (2020)

26. Y. Zhang, R. Sui, Y. Chen, H. Liang, J. Shi, H. Piao, Long noncoding RNA MT1JP inhibits proliferation, invasion, and migration while promoting apoptosis of glioma cells through the activation of PTEN/Akt signaling pathway. J Cell Physiol 234, 19553-19564 (2019)

27. J.M. Engreitz, K. Sirokman, P. McDonel, A.A. Shishkin, C. Surka, P. Russell, S.R. Grossman, A.Y. Chow, M. Guttman, E.S. Lander, RNA-RNA interactions enable specific targeting of noncoding RNAs to nascent pre-mRNAs and chromatin sites. Cell 159, 188199 (2014)

28. G. Yu, Z. Jia, Z. Dou, miR-24-3p regulates bladder cancer cell proliferation, migration, invasion and autophagy by targeting DEDD. Oncol Rep 37, 1123-1131 (2017)

29. J. Liu, Z. Chen, Y. Cui, H. Wei, Z. Zhu, F. Mao, Y. Wang, Y. Liu, Berberine promotes XIAP-mediated cells apoptosis by upregulation of miR-24-3p in acute lymphoblastic leukemia. Aging (Albany NY) 12, 3298-3311 (2020)

30. M.L. Hartman, M. Czyz, BCL-w: Apoptotic and non-apoptotic role in health and disease. Cell Death Dis 11, 260 (2020)

31. S. Bhatlekar, I. Basak, L.C. Edelstein, R.A. Campbell, C.R. Lindsey, J.E. Italiano Jr., A.S. Weyrich, J.W. Rowley, M.T. Rondina, M. Sola-Visner, P.F. Bray, Anti-apoptotic BCL2L2 increases megakaryocyte proplatelet formation in cultures of human cord blood. Haematologica 104, 2075-2083 (2019)

32. N. Zhang, X. Meng, L. Mei, J. Hu, C. Zhao, W. Chen, The long non-coding RNA SNHG1 attenuates cell apoptosis by regulating miR-195 and BCL2-like protein 2 in human Cardiomyocytes. Cell Physiol Biochem 50, 1029-1040 (2018)

33. R. Liu, H. Guo, S. Lu, MiR-335-5p restores cisplatin sensitivity in ovarian cancer cells through targeting BCL2L2. Cancer Med 7, 4598-4609 (2018)

Publisher's note Springer Nature remains neutral with regard to jurisdictional claims in published maps and institutional affiliations. 